\title{
Clinical Efficacy of Intravenous Immunoglobulin for BK Polyomavirus-Associated Nephropathy After Living Kidney Transplantation
}

This article was published in the following Dove Press journal:

Therapeutics and Clinical Risk Management

\author{
Soichi Matsumura' \\ Taigo Kato (iD) ${ }^{1,2}$ \\ Ayumu Taniguchi' \\ Masataka Kawamura' \\ Shigeaki Nakazawa' \\ Tomoko Namba-Hamano iD ${ }^{3}$ \\ Toyofumi Abe' \\ Norio Nonomura (D) \\ Ryoichi Imamura (D) \\ 'Department of Urology, Osaka \\ University Graduate School of Medicine, \\ Osaka 565-087I, Japan; ${ }^{2}$ Department of \\ Urological Immuno-Oncology, Osaka \\ University Graduate School of Medicine, \\ Osaka 565-087I, Japan; ${ }^{3}$ Department of \\ Nephrology, Osaka University Graduate \\ School of Medicine, Osaka 565-087I, \\ Japan
}

Correspondence: Taigo Kato Department of Urology, Osaka University

Graduate School of Medicine, 2-2

Yamadaoka, Suita, Osaka 565-087I, Japan

Tel +8I-6-6879-353I

Fax +8I-6-6879-3534

Email kato@uro.med.oaska-u.ac.jp
Purpose: BK polyomavirus (BKPyV)-associated nephropathy (BKPyVAN) is one of the most difficult infections to be treated after kidney transplantation. Although patients with BKPyVAN usually received a reduction of immunosuppressive agents, the majority of these patients undergo the loss of the graft kidney without any effective treatment afterward. Therefore, development of more effective therapy for BKPyVAN is eagerly expected.

Patients and Methods: Among patients who underwent a kidney transplantation between January 2016 and April 2019 at our hospital, there were five cases of BKPyVAN. After the initial diagnosis, all patients discontinued administration of mycophenolate mofetil (MMF), which was not enough to diminish decoy cells in urine cytology test. Therefore, all patients received additional intravenous immunoglobulin (IVIG) (100 mg/kg/day) therapy for five days and were evaluated for the therapeutic effect of IVIG with immunohistochemical examination using re-biopsy samples of the graft kidney.

Results: After IVIG therapy, 2 cases showed negative decoy cells in urine and 3 cases showed a drastic decrease of plasma BK virus load. Importantly, simian virus (SV) 40 large $\mathrm{T}$ antigens diminished after IVIG administration in all cases, which degraded polyomavirus nephropathy classification.

Conclusion: Although it is difficult to treat BKPyVAN after kidney transplant, IVIG therapy was considered to a promising treatment to improve severity of BKPyVAN especially in cases that dose reduction of immunosuppressive agents was ineffective.

Keywords: BK virus, BK virus nephropathy, intravenous immunoglobulin; IVIG, kidney transplantation, polyomavirus nephropathy classification

\section{Introduction}

In the field of kidney transplantation, advances in immunosuppressants have strikingly reduced the incidence of acute rejection and significantly improved kidney graft survival. However, despites these benefits, long-term use of immunosuppressants sometimes cause infectious diseases as well as malignancies, leading to allograft loss. ${ }^{1}$

Among various types of infection after kidney transplantation, BK polyomavirus (BKPyV) infection is one of the serious issues since BKPyV may accelerate the loss of graft kidney. ${ }^{2} \mathrm{BKPyV}$ is classified into 4 genotypes, I-IV, and genotype I and IV are the most prevalent and widespread worldwide (approximately 95\%). ${ }^{3}$ However, it is unclear which specific BKPyV genotype is related to developing BKPyV-associated nephropathy (BKPyVAN). BKPyV infection after kidney 
transplantation is usually suspected by detecting BKPyV in urine or blood by testing quantitative polymerase chain reaction (PCR). It is reported that urine and plasma $\mathrm{BK}$ viral load by quantitative PCR were positive in 30-40\% and $10-20 \%$ of all kidney transplant recipients, respectively (The positive criterion by quantitative PCR tests is 100 copies $/ \mathrm{mL}$ or more). As a result of histological findings, about 1 to $10 \%$ of all kidney transplant recipients are diagnosed as BKPyVAN by allograft biopsies. $^{4}$ BKPyVAN after kidney transplantation is very difficult to be treated because there is no effective antiviral drug. In general, although clinical guidelines recommend regular monitoring and timely reduction of immunosuppressants for BKPyVAN such as reduction or discontinuation of calcineurin inhibitors and mycophenolate mofetil (MMF), allograft loss occurs in up to $67 \%$ with BKPyVAN. ${ }^{5}$

In this study, we evaluated the efficacy and safety of intravenous immunoglobulin (IVIG) for intractable BKPyVAN in the patients of inadequate response for reduction of immunosuppressants. Interestingly, low-dose IVIG (100 mg/kg/day for 5 days) treatment substantially improved BKPyVAN with compelling evidences of decrease of BKPyV copy numbers in plasma and disappearance of simian virus (SV) 40 large $\mathrm{T}$ antigens. Collectively, IVIG treatment can be the promising option to ameliorate the extent of BKPyVAN immediately.

\section{Materials and Methods}

A total of 112 patients underwent kidney transplantation between January 2016 and April 2019 at our hospital. All organs were donated voluntarily with written informed consent, and the organ donations were conducted in accordance with the Declaration of Istanbul.

For the screening test of BK virus, we first applied urine cytology test, which is covered by Japanese health insurance. Urine cytology test was performed every 3 months in all patients to evaluate the presence of cells containing nuclear viruses (decoy cells) and 14 patients were positive for first screening. Among them, 5 patients with positive decoy cells for two consecutive times were subjected to quantification of plasma BK viral genomic load by quantitative real-time PCR. When the copy numbers in BK Viremia exceeds 200 copies $/ \mathrm{mL}$, the test was considered positive. If BKPyVAN was suspected during tests above, biopsy of graft kidney was performed for definite diagnosis. Finally, these 5 patients were diagnosed as BKPyVAN after kidney transplantation.

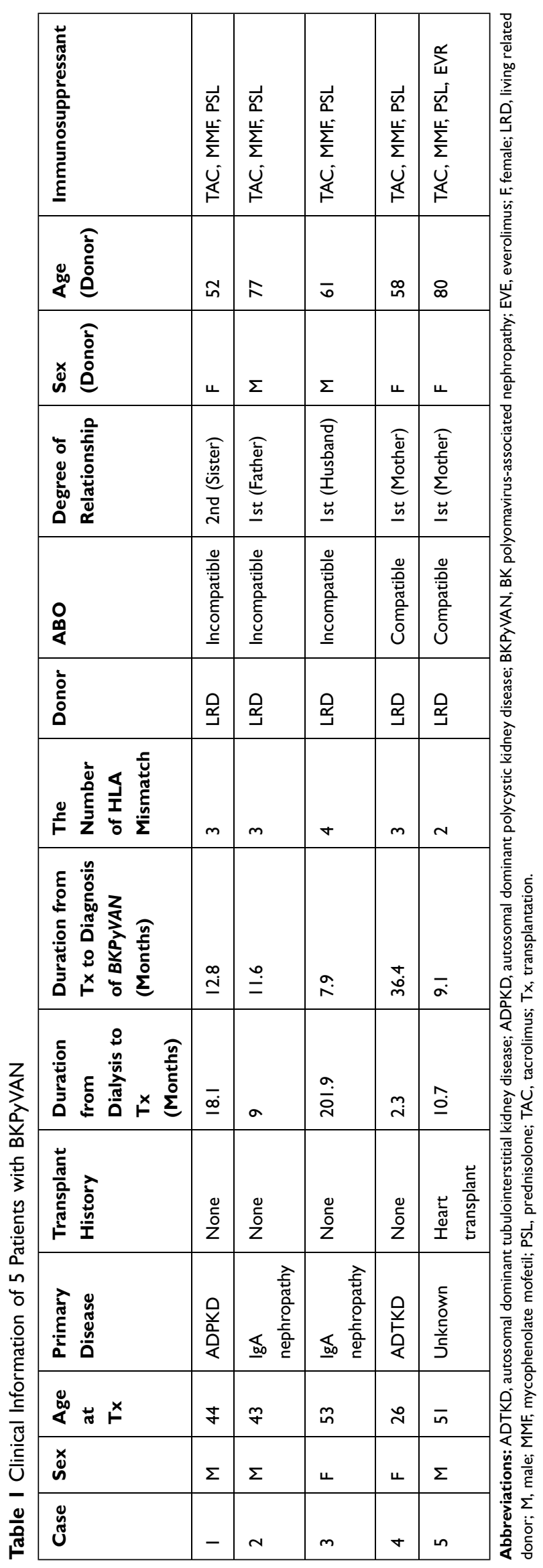


BKPyVAN was defined by positive immunohistochemical nuclear staining using anti-SV40 large $T$ antigen monoclonal antibody. According to polyomavirus nephropathy (PVN) classification from the Banff Working Group, the histological features were classified to PVN class 1, class 2, and class 3 by Banff pvl and ci score. ${ }^{6}$ The pvl scoring is based on the extent of virally induced tubular changes. A tubule with intranuclear viral inclusion bodies (type 1 or 2) and/or a positive SV40 T antigen in one or more cells per tubular cross-section is considered "a positive tubule." The overall percentage of positive tubular cross-sections is estimated in the entire biopsy sample (all available cores, cortex, and medulla): pvl 1: $\leq 1 \%$; pvl 2: $>1 \%$ to $\leq 10 \%$; pvl $3:>10 \%$ of all tubules/ducts with viral replication. The Banff ci Score is also classified by the percentage of interstitial fibrosis. It is defined as ci 0 , ci 1 , ci 2 and ci 3 when the percentage of interstitial fibrosis is $0-5 \%, 5-25 \%, 25-50 \%$ and $50 \%$ or more, respectively. The pathological diagnosis was performed under the same well-experienced pathologist.

After diagnosis of BKPyVAN by graft kidney biopsy, all patients discontinued MMF administration and the decrease of Tacrolimus (TAC) according to clinical guidelines. ${ }^{7}$ In case 4, TAC was converted to Cyclosporine A (CsA). In this study, all patients received IVIG $(100 \mathrm{mg} / \mathrm{kg} /$ day $)$ for 5 days (approved dose in Japanese health insurance) after the dose modification of immunosuppressive agents and received rebiopsy of graft kidney to evaluate the status of BKPyVAN.

\section{Results}

Background of 5 patients' cases are shown in Table 1. All cases received transplant from living related donors. Three of the 5 cases received ABO incompatible kidney transplantation. Four patients were given immunosuppressive agents with TAC, MMF, Prednisolone (PSL) and Basiliximab (BXM) as induction therapies and remaining 1 patient with TAC, MMF, Everolimus (EVR), PSL and BXM. Case 5 already had TAC and MMF since he received heart transplant 11 years before kidney transplant. The median age at transplantation was 44 years (26-53 years) and the median time to diagnosis of BKVN was 11.6 month (7.9-36.4 months). At the diagnosis of BKPyVAN, all cases were classified as class II according to PVN classification (Table 2). BK viremia was considered positive in 3 of the 5 cases (case 1.2 and 3 ).

Urine decoy cells and plasma BK viral genomic load were still remained positive even after the discontinuation of MMF in all cases (Table 2). Therefore, all patients received IVIG treatment at the dose of $100 \mathrm{mg} / \mathrm{kg}$ per day for 5 days. In 4 cases (case 1,3,4 and 5), the

Table 2 Comparison of Decoy Cells in Urine, Plasma BK Viral Load and Pathological Findings at the Time of Pre-Treatment and PostTreatment with IVIG

\begin{tabular}{|c|c|c|c|c|c|c|c|c|c|c|c|c|}
\hline \multirow[t]{3}{*}{ Case } & \multicolumn{5}{|c|}{ At Diagnosis of BKPyVAN } & \multicolumn{2}{|c|}{$\begin{array}{l}\text { Post Reduction of } \\
\text { Immunosuppressants }\end{array}$} & \multicolumn{5}{|c|}{ Post IVIG Treatment } \\
\hline & \multirow{2}{*}{$\begin{array}{l}\text { Decoy } \\
\text { Cells in } \\
\text { Urine }\end{array}$} & \multirow{2}{*}{$\begin{array}{l}\text { Plasma } \\
\text { BK Viral } \\
\text { Load }\end{array}$} & \multicolumn{3}{|c|}{ Pathological Diagnosis } & \multirow{2}{*}{$\begin{array}{l}\text { Decoy } \\
\text { Cells in } \\
\text { Urine }\end{array}$} & \multirow{2}{*}{$\begin{array}{l}\text { Plasma } \\
\text { BK Viral } \\
\text { Load }\end{array}$} & \multirow{2}{*}{$\begin{array}{l}\text { Decoy } \\
\text { Cells in } \\
\text { Urine }\end{array}$} & \multirow{2}{*}{$\begin{array}{l}\text { Plasma } \\
\text { BK Viral } \\
\text { Load }\end{array}$} & \multicolumn{3}{|c|}{ Pathological Diagnosis } \\
\hline & & & $\begin{array}{l}\text { PVN } \\
\text { Class }\end{array}$ & $\begin{array}{l}\text { pvl } \\
\text { Score }\end{array}$ & $\begin{array}{l}\text { ci } \\
\text { Score }\end{array}$ & & & & & $\begin{array}{l}\text { PVN } \\
\text { Class }\end{array}$ & $\begin{array}{l}\text { pvl } \\
\text { Score }\end{array}$ & $\begin{array}{l}\text { ci } \\
\text { Score }\end{array}$ \\
\hline 1 & Positive & 30,000 & Class II & 2 & 1 & Positive & 10,000 & Positive & 300 & $\begin{array}{l}\text { Presum } \\
\text { PVN }\end{array}$ & tive & I \\
\hline 2 & Positive & 8000 & Class II & 2 & 1 & Positive & 70,000 & $\begin{array}{l}\text { Negative } \\
\text { (after } 5 \\
\text { months) }\end{array}$ & $<200$ & $\begin{array}{l}\text { Presum } \\
\text { PVN }\end{array}$ & tive & I \\
\hline 3 & Positive & 5000 & Class II & 2 & 2 & Positive & 200 & $\begin{array}{l}\text { Negative } \\
\text { (after } 5 \\
\text { months) }\end{array}$ & $<200$ & $\begin{array}{l}\text { Class } \\
\text { II }\end{array}$ & I & 3 \\
\hline 4 & Positive & Negative & Class II & 2 & 2 & Positive & Negative & Positive & Negative & $\begin{array}{l}\text { Presum } \\
\text { PVN }\end{array}$ & tive & 2 \\
\hline 5 & Positive & Negative & Class II & 2 & I & Positive & Negative & Positive & Negative & $\begin{array}{l}\text { Presum } \\
\text { PVN }\end{array}$ & tive & I \\
\hline
\end{tabular}

Abbreviation: BKPyVAN, BK polyomavirus-associated nephropathy. 
additional 1 cycle of IVIG was administered due to persistent positive decoy cells or high copies of plasma BK viral genomic load by quantitative PCR after first cycle of IVIG. There were no adverse events related with IVIG administration. After IVIG treatment, decoy cells disappeared in 2 of 5 cases and BK viral load drastically decreased in 3 cases that had high copies of BK virus in plasma (Table 2). The clinical course of typical case (case 3) was shown in Figure 1A. Importantly, SV40positive cells disappeared after IVIG treatment in all cases (Figure 1B), which led to the improvement of PVN class and pvl score (Figure 1C and Table 2). The number of lymphocytes in the interstitium was drastically decreased and the area of interstitial fibrosis after IVIG therapy (The typical pathological findings (case 2) were shown in Figure 1C). There was no evidence for acute rejection in re-biopsy samples.

\section{Discussion}

BKPyV infection in transplant recipients has become one of the critical issues with the widespread of potent immunosuppressive agents. The infection may cause significant deleterious effects such as BKPyV which cause BKPyVAN in kidney transplant and hemorrhagic cystitis

A
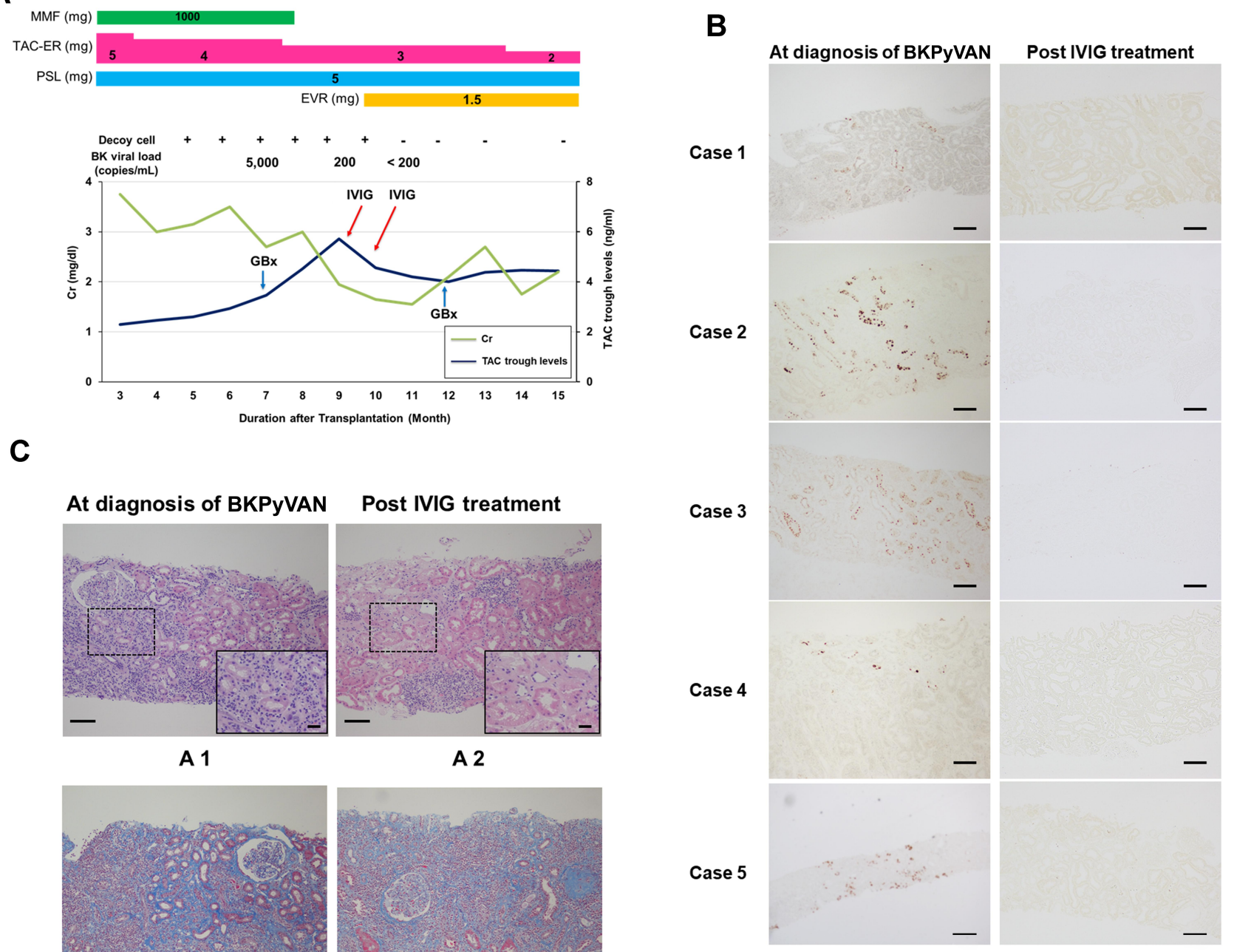

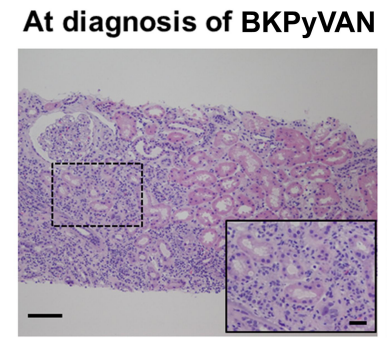

A 1

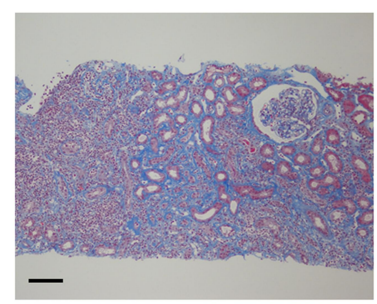

B 1

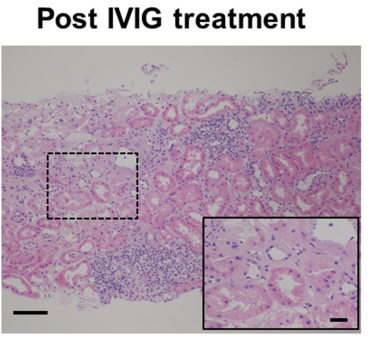

A 2

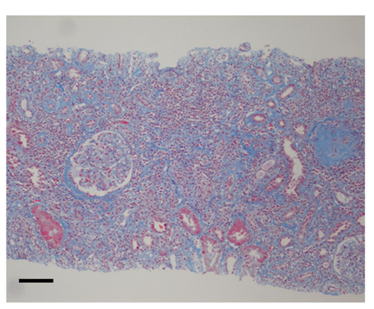

B 2

Figure I Comparison of representative renal histopathological findings at the time of initial biopsy and repeated biopsy after IVIG therapy. (A) Clinical course of typical case (case 3). (B) SV40 positive cells diminished after IVIG therapy in all cases. (C) Many intranuclear inclusions were observed and lymphocytes infiltrated into the interstitium (AI). After IVIG therapy, the inclusion bodies in the nucleus disappeared. The number of lymphocytes was decreased (A2). A wide range of interstitial fibrosis was observed in the sample of initial diagnosis (BI). The area of interstitial fibrosis improved after IVIG therapy (B2). HE staining for AI and A2 and Elastica-Masson staining for BI and B2. Magnification: $100 \times$ for all pictures, $400 \times$ for inset pictures of $A 1$ and $A 2$. Scale bars $=100 u m$.

Abbreviations: BKPyVAN, BK polyomavirus-associated nephropathy; IVIG, intravenous immunoglobulin. 
in hematopoietic stem cell transplant recipients. ${ }^{8,9}$ So far, as a treatment for BKPyVAN, first it is necessary to reduce the dose of calcineurin inhibitors and MMF. ${ }^{5,10}$ For a certain number of patients, conversion of TAC to lowdosage CsA is effective to lower the severity of BKPyVAN since the immunosuppressive strength of CsA is weaker than that of TAC. ${ }^{11}$ Anti-viral drugs, leflunomide or cidofovir had a certain effect on BKPyVAN. ${ }^{12}$ However, it is difficult to use these drugs since these are nephrotoxic for patients with unstable renal function and have low selectivity for BKPyV. Given these findings, the new approach to treat BKPyVAN is eagerly expected in clinical settings.

IVIG is a pool of IgG from thousands of healthy donors who experienced various types of infectious disease and has been used to treat patients with autoimmune and chronic inflammatory diseases, such as Kawasaki disease, multiple sclerosis, and lupus. ${ }^{13}$ Commercially available IVIG also contains virus neutralizing antibodies against all major genotypes of $\mathrm{BKPyV} .^{14,15} \mathrm{Vu}$ et al reported high-dose of IVIG $(1.0 \mathrm{~g} / \mathrm{kg})$ in combination with leflunomide for two times significantly decreased BK viral load within 1 month of treatments. ${ }^{16}$ In many cases, allograft biopsies after IVIG therapy did not show any evidence of rejection or BKPyVAN. In our study, we evaluated the efficacy and safety of much lower dose of IVIG $(100 \mathrm{mg} / \mathrm{kg} / \mathrm{day}$ for 5 days, $500 \mathrm{mg} / \mathrm{kg}$ in total) compared to the dose of previous reports as the additional therapy for BKPyVAN patients who had less response for reducing immunosuppressive agents. ${ }^{16}$

Surprisingly, in our cohort, we found that the treatment of lower dose of IVIG was enough to diminish SV40 large $T$ antigen and degrade PVN class (Presumptive PVN) in all patients. Considering that PVN class significantly affects renal function and the decrease of rate of allograft loss, the degradation of PVN class with IVIG therapy may have the benefits for long-term prognosis. ${ }^{6}$

On the contrary, the level of serum creatine level improved in only 1 patient at 6 months after IVIG treatment with the evidence that ci score still remained unchanged at post-IVIG treatment samples. Given that the improvement of renal function took at least 12 months after high-dose IVIG therapy, we need to observe the patients of our cohort over a longer period. ${ }^{16}$

Some limitations should be considered when interpreting these results. First, the sample size was small due to the short duration of inclusion criteria. Second, first screening of urine cytology tests for BKPyV might delay the diagnosis of BKPyVAN in allograft biopsy. We hope the measurement of plasma or urine BK viral load will be permitted in Japanese health insurance as the first screening tool. Third, this was a single-arm study to evaluate IVIG therapy for BKPyV infection and other-arm without IVIG will be needed. We have a plan to compare these 2 arms with randomized trials to provide firm evidence of additional IVIG therapy for BKPyVAN in kidney transplantation.

\section{Conclusion}

IVIG therapy appears to be effective in the treatment of BKPyVAN and can prevent deteriorating renal function, which may avoid allograft loss and improve long-term graft survival.

\section{Ethical Approval}

The study protocol was approved by the Institutional Review Board of Osaka University (approval number 668-5). All patients provided written informed consents.

All procedures and the use of tissues were performed in accordance with the Declaration of Helsinki principles.

\section{Acknowledgments}

We thank Atsuko Yasumoto for technical assistance.

\section{Disclosure}

The authors report no conflicts of interest for this work.

\section{References}

1. Meier-Kriesche HU, Schold JD, Srinivas TR, Kaplan B. Lack of improvement in renal allograft survival despite a marked decrease in acute rejection rates over the most recent era. Am J Transplant. 2004;4 (3):378-383. doi:10.1111/j.1600-6143.2004.00332.x

2. Kable K, Davies CD, O'connell PJ, Chapman JR, Nankivell BJ. Clearance of BK virus nephropathy by combination antiviral therapy with intravenous immunoglobulin. Transplant Direct. 2017;3(4):e142. doi:10.1097/TXD.0000000000000641

3. Wunderink HF, De Brouwer CS, Gard L, et al. Source and relevance of the BK polyomavirus genotype for infection after kidney transplantation. Open Forum Infect Dis. 2019;6(3):ofz078. doi:10. 1093/ofid/ofz078

4. Hirsch HH, Randhawa P. Practice AIDCo. BK polyomavirus in solid organ transplantation. Am J Transplant. 2013;13(Suppl 4):179-188. doi:10.1111/ajt.12110

5. Saad ER, Bresnahan BA, Cohen EP, et al. Successful treatment of BK viremia using reduction in immunosuppression without antiviral therapy. Transplantation. 2008;85(6):850-854. doi:10.1097/TP.0b013e 318166cba8

6. Nickeleit V, Singh HK, Randhawa P, et al. The Banff Working Group classification of definitive polyomavirus nephropathy: morphologic definitions and clinical correlations. J Am Soc Nephrol. 2018;29 (2):680-693. doi:10.1681/ASN.2017050477 
7. Hirsch HH, Randhawa P. Practice ASTIDCo. BK virus in solid organ transplant recipients. Am J Transplant. 2009;9(Suppl 4):S136-146. doi:10.1111/j.1600-6143.2009.02904.x

8. Hirsch HH, Brennan DC, Drachenberg CB, et al. Polyomavirusassociated nephropathy in renal transplantation: interdisciplinary analyses and recommendations. Transplantation. 2005;79(10):1277-1286. doi:10.1097/01.tp.0000156165.83160.09

9. Dropulic LK, Jones RJ. Polyomavirus BK infection in blood and marrow transplant recipients. Bone Marrow Transplant. 2008;41 (1):11-18. doi:10.1038/sj.bmt.1705886

10. Bischof N, Hirsch $\mathrm{HH}$, Wehmeier C, et al. Reducing calcineurin inhibitor first for treating BK polyomavirus replication after kidney transplantation: long-term outcomes. Nephrol Dial Transplant. 2019;34(7):1240-1250. doi:10.1093/ndt/gfy346

11. Chen XT, Li J, Deng RH, et al. The therapeutic effect of switching from tacrolimus to low-dose cyclosporine A in renal transplant recipients with BK virus nephropathy. Biosci Rep. 2019;39(2). doi:10.1042/BSR20182058

12. Lamarche $\mathrm{C}$, Orio $\mathrm{J}$, Collette $\mathrm{S}$, et al. BK polyomavirus and the transplanted kidney: immunopathology and therapeutic approaches. Transplantation. 2016;100(11):2276-2287. doi:10.1097/TP.0000000 000001333
13. Jolles S, Sewell WA, Misbah SA. Clinical uses of intravenous immunoglobulin. Clin Exp Immunol. 2005;142(1):1-11. doi:10.1111/j.1365-2249.2005.02834.x

14. Randhawa P, Pastrana DV, Zeng G, et al. Commercially available immunoglobulins contain virus neutralizing antibodies against all major genotypes of polyomavirus BK. Am J Transplant. 2015;15 (4):1014-1020. doi:10.1111/ajt.13083

15. Pastrana DV, Brennan DC, Cuburu N, et al. Neutralization serotyping of BK polyomavirus infection in kidney transplant recipients. PLoS Pathog. 2012;8(4):e1002650. doi:10.1371/journal.ppat. 1002650

16. Vu D, Shah T, Ansari J, Naraghi R, Min D. Efficacy of intravenous immunoglobulin in the treatment of persistent $\mathrm{BK}$ viremia and BK virus nephropathy in renal transplant recipients. Transplant Proc. 2015;47(2):394-398. doi:10.1016/j.transproceed. 2015.01.012

\section{Publish your work in this journal}

Therapeutics and Clinical Risk Management is an international, peerreviewed journal of clinical therapeutics and risk management, focusing on concise rapid reporting of clinical studies in all therapeutic areas, outcomes, safety, and programs for the effective, safe, and sustained use of medicines. This journal is indexed on PubMed Central, CAS,
EMBase, Scopus and the Elsevier Bibliographic databases. The manuscript management system is completely online and includes a very quick and fair peer-review system, which is all easy to use. Visit http://www.dovepress.com/testimonials.php to read real quotes from published authors. 